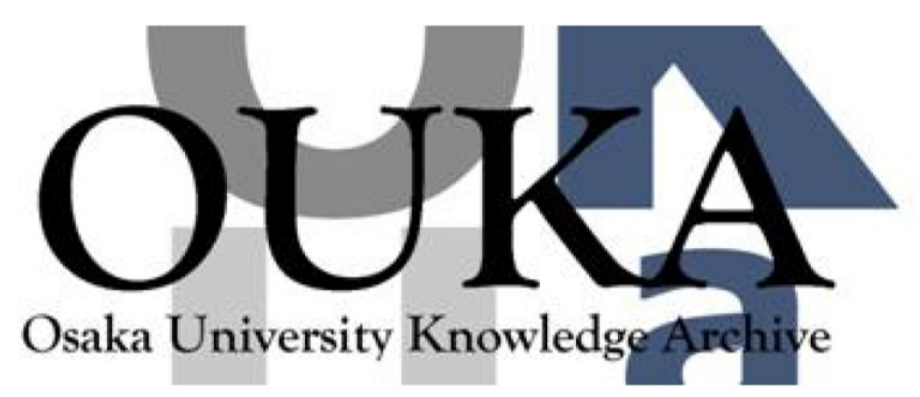

\begin{tabular}{|c|c|}
\hline Title & $\begin{array}{l}\text { Elastic stiffness of metallic multilayers } \\
\text { studied by picosecond ultrasonics: Improved } \\
\text { interpretation of interface elasticity }\end{array}$ \\
\hline Author(s) & $\begin{array}{l}\text { Nakamura, Nobutomo; Yokomura, Ryo; Takeuchi, } \\
\text { Nobutaka et al. }\end{array}$ \\
\hline Citation & $\begin{array}{l}\text { Japanese Journal of Applied Physics. 58(7) } \\
\text { p. } 075504\end{array}$ \\
\hline Issue Date & $2019-06-24$ \\
\hline oaire:version & AM \\
\hline URL & https://hdl. handle. net/11094/84483 \\
\hline rights & $\begin{array}{l}\text { (- } 2019 \text { The Japan Society of Applied Physics. } \\
\text { This Accepted Manuscript is avai lable for reuse } \\
\text { under a Creative Commons Attribution- } \\
\text { NonCommercial-NoDerivatives } 4.0 \text { International } \\
\text { License after the } 12 \text { month embargo period } \\
\text { provided that all the terms of the license are } \\
\text { adhered to. }\end{array}$ \\
\hline Note & \\
\hline
\end{tabular}

Osaka University Knowledge Archive : OUKA

https://ir. Library. osaka-u. ac. jp/

Osaka University 


\title{
Elastic stiffness of metallic multilayers studied by picosecond ultrasonics: improved interpretation of interface elasticity
}

\author{
Nobutomo Nakamura ${ }^{1 *}$, Ryo Yokomura ${ }^{1}$, Nobutaka Takeuchi ${ }^{1}$, Daiki Yamakado ${ }^{1}$, and \\ Hirotsugu Ogi ${ }^{2}$ \\ ${ }^{1}$ Graduate School of Engineering Science, Osaka University, Toyonaka, Osaka 560-8531, Japan \\ ${ }^{2}$ Graduate School of Engineering, Osaka University, Suita, Osaka 565-0871, Japan
}

In metallic multilayers, elastic stiffness is often reported to decrease with increasing number of interfaces. This phenomenon is generally attributed to softening at interfaces, and a decrease of $50 \%$ or more in interface stiffness is expected. This interpretation of elastic stiffness of metallic multilayers is commonly accepted; however, we propose that the decrease of the interface stiffness is doubtful because it is difficult to explain the large decrease using existing models. In the present study, to confirm the validity of the decrease of the interface stiffness, longitudinal-wave elastic stiffness in the stacking direction is measured using picosecond ultrasonics. The elastic stiffness of $\mathrm{Cr} / \mathrm{Fe}, \mathrm{Cu} / \mathrm{Co}$, and $\mathrm{Nb} / \mathrm{Cu}$ multilayers with several thickness ratios is measured, and the decrease in multilayer stiffness is experimentally demonstrated to arise from the decrease in the stiffness of each layer, not from a decrease in interface stiffness.

*E-mail: nobutomo@me.es.osaka-u.ac.jp 


\section{Introduction}

Elastic stiffness of layered structures is generally derived from elastic stiffness and thickness of constituting layers using a simple rule of mixture. However, in metallic multilayer films like superlattice, thickness of each layer becomes of the order of $10^{-9} \mathrm{~m}$ and the number of interfaces increases. In the films, it is supposed that elastic stiffness cannot be derived simply from the stiffness and thickness of constituting layers and stiffness at interfaces has to be taken into account. The interface stiffness has attracted attentions and it has been investigated by several researchers.

In previous studies on elastic stiffness of multilayer films, Rayleigh-wave velocity and the relevant elastic stiffness, ${ }^{1-6)}$ Young's modulus, ${ }^{7)}$ the longitudinal-wave elastic stiffness in the out-of-plane direction, ${ }^{8-12)}$ and components of the elastic stiffness tensor ${ }^{13-17)}$ were measured, and interface stiffness was often deduced from relationship between the number of interface (bilayer thickness) and the measured stiffness. In the studies, elastic stiffness often decreased with decreasing bilayer thickness (increasing the number of interfaces). This indicates that interfaces are softer than the constituting materials; for example, in $\mathrm{Nb} / \mathrm{Cu}$ multilayers, the stiffness decreased by $35 \%$ with the increasing number of interfaces. ${ }^{1)}$ After these studies, it has been accepted that elastic stiffness at interface is different from those of constituting materials. However, if a decrease in interface stiffness was the cause, a reduction greater than $50 \%$ in interface stiffness would be required to explain the observed $35 \%$ decrease. Such a decrease in interfacial stiffness is unrealistic. The grain-boundary superlattice model ${ }^{18)}$ is often considered as a possible cause of the interface softening. This model explains the interface softening using strain dependence of elastic stiffness. Using strain dependence of monolayer film stiffness deduced from strain dependence of bulk stiffness, ${ }^{19)}$ the $50 \%$-stiffness change in the thickness direction is performed when the films are biaxially stretched in the plane direction by $8 \%$ and $20 \%$ for $\mathrm{Ag}$ and $\mathrm{Cu}$ films, respectively. It is doubtful whether the large elastic strain exists at interfaces in metallic multilayers. In addition, this aforementioned phenomenon has not been observed in all of the related studies. For example, in $\mathrm{Cu} / \mathrm{Co}$ multilayers both softening ${ }^{5)}$ and nonsoftening ${ }^{20)}$ have been observed with decreasing bilayer thickness. For these reasons, we consider that elastic stiffness at interfaces is not understood completely although it has been investigated by several researchers.

The purpose of the present study is to identify whether the interface softening exists or not in metallic multilayers using picosecond ultrasonics. ${ }^{21)}$ The Brillouin scattering is a typical method for measuring elastic stiffness of thin films. This can measure elastic stiffness without 
touching a specimen, but substrate stiffness is required to extract the multilayer stiffness, and some components of the elastic stiffness tensor can be determined by using the dispersion relationship. ${ }^{13-17)}$ In contrast, to simplify the analysis, the elastic character of a substrate is sometimes assumed to be isotropic even if it is anisotropic. ${ }^{14)}$ Such assumptions lead to an uncertainty in the measured stiffness. By using free-standing multilayers removed from substrate, ${ }^{17)}$ elastic stiffness can be determined without using the substrate stiffness. However, multilayers consisting of more than a thousand layers are needed for the measurement. In the multilayers, coherency at the interface may be disturbed. In this study, we use picosecond ultrasonics. The round-trip time of acoustic waves propagating in the thickness direction of a thin film is measured using the pump-probe technique, and the longitudinal-wave elastic stiffness in the thickness direction is determined from it. Only mass density and film thickness are the needed parameters, which is an advantage over other methods. In addition, the longitudinal-wave elastic stiffness directly reflects the binding force at interfaces, and it is sensitive to interface stiffness (see Appendix). Furthermore, this method can measure the elastic stiffness of multilayers thinner than $100 \mathrm{~nm}$. For these reasons, this method has been applied to several metallic films ${ }^{19,22-24)}$ and multilayers, ${ }^{8-12,25-27)}$ and it is recognized as a powerful tool for studying the elastic stiffness of thin films. Regarding metallic multilayers, softening behavior at interfaces is reported ${ }^{8-10)}$ as observed in the Brillouin scattering. However, in multilayers showing substantial softening, long-range crystalline order was not observed and disordered structure within the elemental layer was observed by the X-ray diffraction, ${ }^{8}$ which implies that the interface softening may not be the dominant cause.

In multilayers, lattice misfit at interfaces causes a strain filed, and it varies depending on thickness ratio of layers. If the softening originates from the strain effect at interfaces, degree of the softening will vary depending on the thickness ratio. In previous studies, thickness ratio was usually fixed and bilayer thickness was varied. Therefore, we consider that further investigation on multilayer systems with various thickness ratios is needed to conclude that softening happens at interfaces. For these reasons, we prepare $\mathrm{Cr} / \mathrm{Fe}, \mathrm{Cu} / \mathrm{Co}$, and $\mathrm{Nb} / \mathrm{Cu}$ multilayers comprehensively with various thickness ratios, and their elastic stiffness is measured. Structure of all multilayers is evaluated using the X-ray reflectivity and X-ray diffraction measurements. Finally, we demonstrate that the elastic stiffness of multilayers is lowered by the softening of each layer, not by the softening at interfaces. 


\section{Experimental}

Multilayers were deposited onto single-crystal Si substrates by radio frequency magnetron sputtering. The thickness of each layer was controlled by changing the deposition time. In $\mathrm{Cr} / \mathrm{Fe}$ multilayers, the thickness of $\mathrm{Fe}\left(d_{\mathrm{Fe}}\right)$ was 1 or $5 \mathrm{~nm}$ and that of $\mathrm{Cr}\left(d_{\mathrm{Cr}}\right)$ was varied from 0.6 to $9.3 \mathrm{~nm}$. The number of bilayers $n$ was varied so that the total thickness $d$ was approximately $60 \mathrm{~nm}$. In $\mathrm{Cu} / \mathrm{Co}$ multilayers, the thickness of Co layer $\left(d_{\mathrm{Co}}\right)$ was $1 \mathrm{~nm}$ and that of $\mathrm{Cu}\left(d_{\mathrm{Cu}}\right)$ was varied from 0.5 to $2.1 \mathrm{~nm}$. The total thickness was approximately 40, 60 , or $90 \mathrm{~nm}$. In $\mathrm{Nb} / \mathrm{Cu}$ multilayers, the thickness of $\mathrm{Nb}\left(d_{\mathrm{Nb}}\right)$ was 1,3 , or $5 \mathrm{~nm}$ and $d_{\mathrm{Cu}}$ was varied from 0.6 to $10 \mathrm{~nm}$. A series of specimens with $d_{\mathrm{Nb}} / d_{\mathrm{Cu}}=1$ was also prepared, in which $d_{\mathrm{Nb}}+d_{\mathrm{Cu}}$ is in the range of 1 to $22 \mathrm{~nm}$. The total thickness of the $\mathrm{Nb} / \mathrm{Cu}$ multilayers was approximately $60 \mathrm{~nm}$. The total thickness of the multilayers $(d)$ was determined using X-ray reflectivity measurements, and the multilayer structure was confirmed using X-ray diffraction analysis.

We define a Cartesian coordinate system in which the $x_{3}$-axis is parallel to the stacking direction and the $x_{1}$ - and $x_{2}$-axes are parallel to the substrate surface. In picosecond ultrasonics, we excite an acoustic pulse by irradiating the multilayer surface with a pulse laser whose wavelength is $800 \mathrm{~nm}$. The acoustic pulse propagates in the $x_{3}$ direction and partially reflects at the interface between the multilayer and the substrate. The reflected pulse arrives at the multilayer surface, and it changes the light reflectivity. We irradiate the multilayer surface with another time-delayed pulse laser whose wavelength is $400 \mathrm{~nm}$, and measure the reflectivity change on the surface. By measuring the reflectivity change with different delay times, the multiple reflection of the longitudinal acoustic pulse within the multilayer film is detected. From the round-trip time $\Delta t$, the elastic stiffness $C_{\perp}\left(=C_{33}\right)$ of the multilayer is determined, $C_{\perp}=\rho(2 d / \Delta t)^{2}$, where $\rho$ is the averaged mass density of the multilayer calculated from the reported mass densities of bulk materials of individual layers. ${ }^{28-31)}$ In the following, the elastic stiffness of the multilayer is described as macroscopic elastic stiffness to distinguish it from elastic stiffness of each layer. For comparison, monolayer $\mathrm{Cu}, \mathrm{Nb}, \mathrm{Fe}$, and $\mathrm{Cr}$ films with $d \approx 60 \mathrm{~nm}$ were prepared and their elastic stiffness was measured.

\section{Results}

Figure 1 shows typical X-ray reflectivity spectra. In the spectra of all of the multilayers, oscillation patterns with small period are observed. The patterns originate from interferences between X-rays reflected at the top surface of the multilayer and the interface with the substrate, and their periodicity depends on the total thickness $d$ of the multilayer. By fitting a 
theoretical curve ${ }^{32)}$ to the patterns, the total thickness was determined. In the spectra, broad peaks are also observed. The peaks originate from interferences between X-rays reflected at the interfaces between layers in the multilayer, and its appearance confirms the periodic multilayer structure. Figure 2 shows typical X-ray diffraction patterns. In the $\mathrm{Cr} / \mathrm{Fe}$ multilayers, a diffraction peak is observed. Its diffraction angle is close to those from $\mathrm{Cr}(110)$ and $\mathrm{Fe}(110)$ planes, and it indicates that $\mathrm{Cr}(110)$ and $\mathrm{Fe}(110)$ planes are predominantly aligned parallel to the film surface. A similar behavior is observed in the patterns of the $\mathrm{Cu} / \mathrm{Co}$ multilayers, where $\mathrm{Cu}(111)$ and $\mathrm{Co}(111)$ planes are aligned parallel to the multilayer surface. Regarding Co layers, its crystallographic structure, hcp or fcc, is not identified from the X-ray diffraction spectra, because diffraction angles of hcp-Co (002) and fcc-Co(111) are close to each other. In a previous study, it is reported that Co film shows fcc structure when it is very thin. ${ }^{33)}$ Referring to the study, we assume that structure of Co layers is fcc. This assumption hardly affects the following discussion. In the patterns of the $\mathrm{Nb} / \mathrm{Cu}$ multilayers, diffraction peaks including satellite peaks are observed at $d_{\mathrm{Cu}} \geq 1.0 \mathrm{~nm}$, and $\mathrm{Nb}(110)$ and $\mathrm{Cu}(111)$ planes are aligned parallel to the multilayer surface. Below this thickness, peaks broaden with decreasing $d_{\mathrm{Cu}}$, which indicates that the crystallographic structure is disordered. Thus, in the multilayers close-packed planes of constituting materials are predominantly aligned parallel to the multilayer surface. In the case of monolayer films, we observed that the close-packed planes were predominantly stacked in the thickness direction, showing the similar crystallographic orientations to the multilayers. From these results, we assume that the close-packed planes are aligned completely parallel to the film surface in multilayer and monolayer films in following discussion.

Figure 3 shows typical pulse-echo signals obtained by picosecond ultrasonics. Multiple echoes originating from a longitudinal acoustic pulse propagating in the thickness direction are observed. At least three echoes are identified, and $\Delta t$ was determined from their time intervals. High-frequency oscillations originating from the localized vibrations in multilay$\mathrm{ers}^{26,34)}$ were not observed in the present study. It is attributed to small differences in the acoustic impedances of composing metals. The pulse-echo measurement was performed at a few different points for each multilayer, and the measurement error in the elastic stiffness was calculated from its standard deviation.

\section{Discussion}

Here, we compare the measured stiffness of the multilayers to the macroscopic elastic stiffness calculated from the bulk stiffness and the film stiffness of elemental materials. Table I 




Fig. 1. Representative $\mathrm{X}$-ray reflectivity spectra. $\mathrm{CoK} \alpha$ radiation was used for $\mathrm{Cr} / \mathrm{Fe}$ and $\mathrm{Nb} / \mathrm{Cu}$ multilayers, whereas $\mathrm{CuK} \alpha$ radiation was used for $\mathrm{Cu} / \mathrm{Co}$ multilayers.

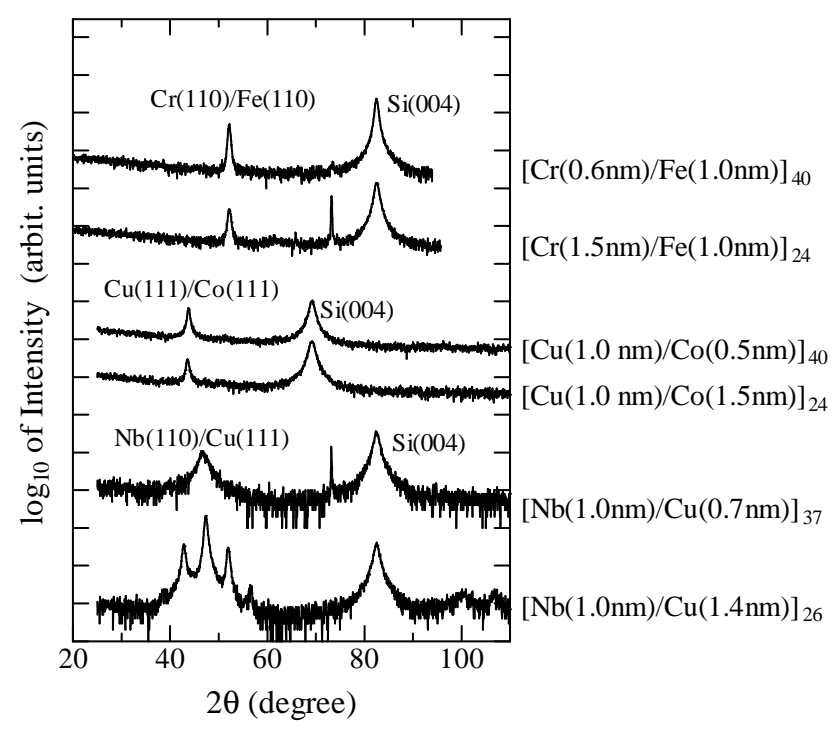

Fig. 2. Representative $\mathrm{X}$-ray diffraction patterns. $\mathrm{CoK} \alpha$ radiation was used for $\mathrm{Cr} / \mathrm{Fe}$ and $\mathrm{Nb} / \mathrm{Cu}$ multilayers, whereas $\mathrm{CuK} \alpha$ radiation was used for $\mathrm{Cu} / \mathrm{Co}$ multilayers. The peaks at $73.2^{\circ}$ denote the $\operatorname{Si}(004)-\operatorname{CoK} \beta$.

summarizes the bulk stiffness and the film stiffness. Regarding bulk stiffness, the stiffness was calculated from the elastic stiffness of single crystals ${ }^{28,30,35-37)}$ assuming that the [111] axis of $\mathrm{Cu}$ and $\mathrm{Co}$ and [110] axis of $\mathrm{Fe}, \mathrm{Cr}$, and $\mathrm{Nb}$ are aligned normal to the film plane, and following equations were used; $C_{[111]}=\left(C_{11}^{\prime}+2 C_{12}^{\prime}+4 C_{44}^{\prime}\right) / 3$ and $C_{[110]}=\left(C_{11}^{\prime}+C_{12}^{\prime}+2 C_{44}^{\prime}\right) / 2$, where $C_{i j}^{\prime}$ is elastic stiffness of single crystals. Regarding film stiffness, the elastic stiffness 


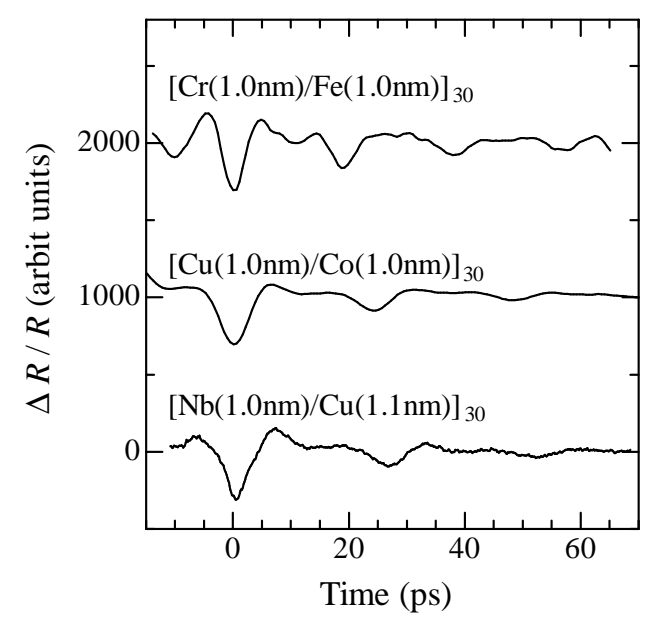

Fig. 3. Representative pulse-echo signals obtained by picosecond ultrasonics. The horizontal axis of the data is shifted such that the first echo appears at 0 ps.

Table I. Elastic stiffness (GPa) of bulk materials and monolayer films.

\begin{tabular}{lcc}
\hline \hline & Bulk & Film \\
\hline $\mathrm{Cu}$ & $235.8^{\mathrm{a}}$ & 200.4 \\
$\mathrm{Co}$ & $366.8^{\mathrm{b}}$ & $279.2^{\mathrm{c}}$ \\
$\mathrm{Fe}$ & $299.5^{\mathrm{d}}$ & 265.6 \\
$\mathrm{Cr}$ & $309.7^{\mathrm{e}}$ & 310.6 \\
$\mathrm{Nb}$ & $211.1^{\mathrm{f}}$ & 216.9 \\
\hline \hline
\end{tabular}

a Calculated from single crystal stiffness in Ref. ${ }^{28)}$

b Ref. ${ }^{35)}$

c Average of film stiffness reported in Ref. ${ }^{38)}$ Film thickness is in the rage of 20 to $63 \mathrm{~nm}$.

d Calculated from single crystal stiffness in Ref. ${ }^{36)}$

e Calculated from single crystal stiffness in Ref. ${ }^{37)}$

f Calculated from single crystal stiffness in Ref. ${ }^{30)}$

values measured by picosecond ultrasonics in the present study are listed; our previous experimental result regarding a $\mathrm{Co} \mathrm{film}^{38)}$ is also shown. The measured film stiffness is smaller than the corresponding bulk stiffness in $\mathrm{Cu}, \mathrm{Co}$, and $\mathrm{Fe}$, and it is approximately the same as the bulk stiffness in $\mathrm{Cr}$ and $\mathrm{Nb}$. Given that disordered structures and excess defects (dislocations and voids) can be formed during deposition, smaller or comparable stiffness is reasonable; we observed similar results in our previous studies on other monolayer films. ${ }^{38-40)}$ Using the simple rule of mixture, the macroscopic stiffness of a multilayer comprising layers A and B is expressed as $\bar{C}_{\perp}=\left(d_{\mathrm{A}}+d_{\mathrm{B}}\right) /\left(d_{\mathrm{A}} / C_{\mathrm{A}}+d_{\mathrm{B}} / C_{\mathrm{B}}\right)$, where $d_{i}$ and $C_{i}$ are the thickness and elastic stiffness of layer $i$, respectively. 


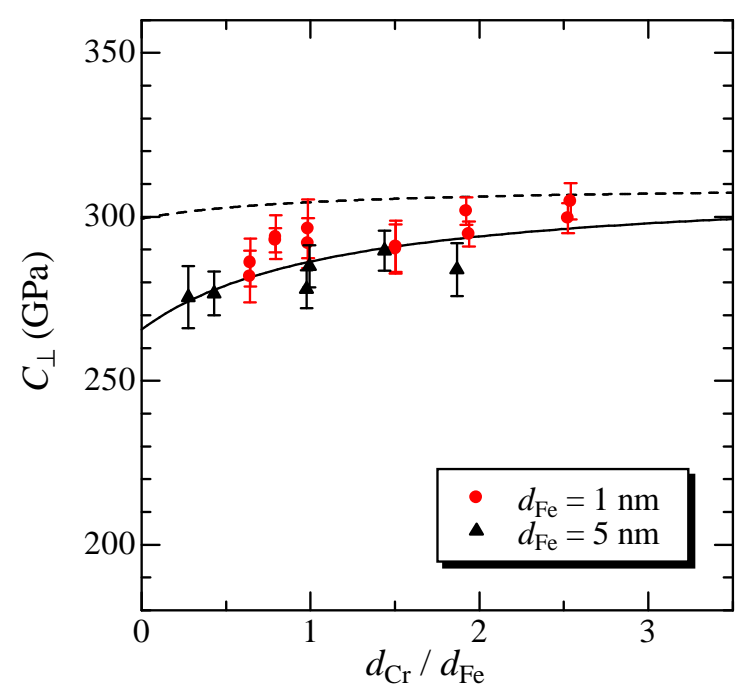

Fig. 4. Elastic stiffness of $\mathrm{Cr} / \mathrm{Fe}$ multilayers. The dashed curve denotes the elastic stiffness calculated from the bulk stiffness. The solid curve denotes the elastic stiffness calculated from the monolayer-film stiffness.

Figure 4 shows the elastic stiffness of $\mathrm{Cr} / \mathrm{Fe}$ multilayers. The measured stiffness decreases with decreasing thickness ratio $d_{\mathrm{Cr}} / d_{\mathrm{Fe}}$, whose behavior is similar to that of the stiffness calculated from the bulk stiffness. However, the measured stiffness is smaller than the stiffness calculated from the bulk stiffness, and is similar to the stiffness calculated from the film stiffness. These behaviors are observed independently of $d_{\mathrm{Fe}}$.

Figure 5 shows the elastic stiffness of $\mathrm{Cu} / \mathrm{Co}$ multilayers. As observed in $\mathrm{Cr} / \mathrm{Fe}$, the measured stiffness is smaller than the stiffness calculated from the bulk stiffness, and it agrees with the stiffness calculated from the film stiffness. These behaviors are observed for all of the multilayers with different total thicknesses; no notable decrease in elastic stiffness is observed.

Figure 6 shows the elastic stiffness of $\mathrm{Nb} / \mathrm{Cu}$ multilayers. The measured elastic stiffness is smaller than the stiffness calculated from the bulk stiffness, and is similar to the stiffness calculated from the film stiffness. This behavior is independent of $d_{\mathrm{Nb}}$, and a series of multilayers with $d_{\mathrm{Cu}} / d_{\mathrm{Nb}}=1$ shows a similar behavior.

In all of the multilayers, the elastic stiffness is smaller than the values calculated from the bulk stiffness, and the measured stiffness agrees with the calculations based on the monolayerfilm stiffness. If each layer is softer than its bulk material, the observed softening is consistently explained without considering the softening at interfaces. To evaluate the effect of the stiffness at the interface, we plotted the relationship between the elastic stiffness and the bilayer thickness in $\mathrm{Nb} / \mathrm{Cu}$ multilayers; the result is shown in Fig. 7, where the measured 


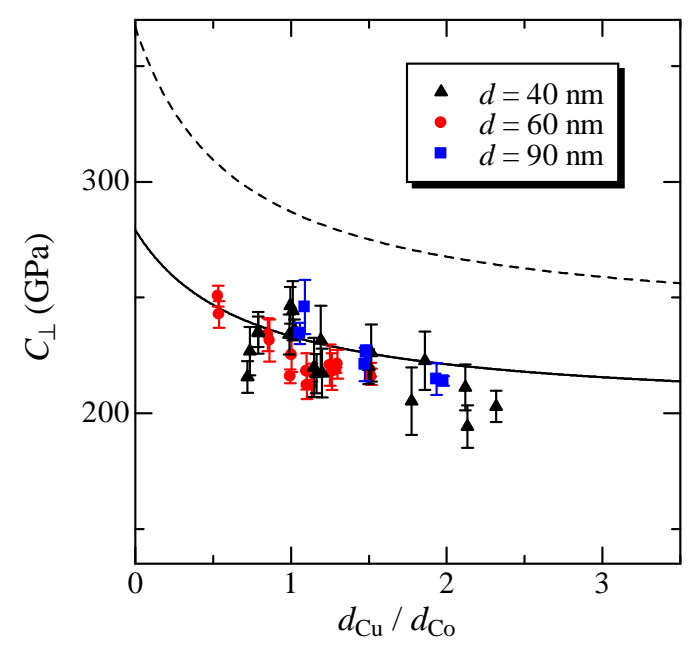

Fig. 5. Elastic stiffness of $\mathrm{Cu} / \mathrm{Co}$ multilayers. The dashed curve denotes the elastic stiffness calculated from the bulk stiffness. The solid curve denotes the elastic stiffness calculated from the monolayer-film stiffness.

Thickness of Co layer is $1 \mathrm{~nm}$.

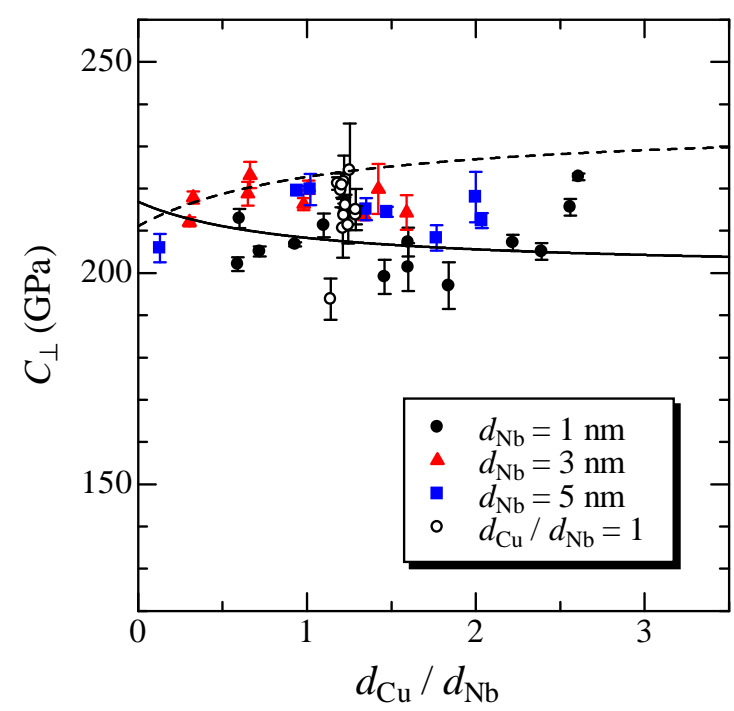

Fig. 6. Elastic stiffness of $\mathrm{Nb} / \mathrm{Cu}$ multilayers. The dashed curve denotes the elastic stiffness calculated from the bulk stiffness. The solid curve denotes the elastic stiffness calculated from the monolayer-film stiffness.

stiffness is normalized by the stiffness calculated from the monolayer-film stiffness $\bar{C}_{\perp}^{\text {Film }}$. In previous studies, ${ }^{1,2)}$ as bilayer thickness was decreased, the elastic stiffness decreased by approximately $35 \%$. In the present results, the elastic stiffness decreases slightly with decreasing bilayer thickness. As observed in the X-ray diffraction analysis, the structure becomes disordered below $d_{\mathrm{Cu}}+d_{\mathrm{Nb}}=2 \mathrm{~nm}$. When the specimens whose bilayer thickness is larger than $2 \mathrm{~nm}$ are considered, the elastic stiffness decreases by approximately $6.5 \%$ with decreasing 




Fig. 7. Elastic stiffness of $\mathrm{Nb} / \mathrm{Cu}$ multilayers. The elastic stiffness is normalized by the stiffness calculated from the monolayer-film stiffness. The solid curve is a guide for the eyes.

bilayer thickness. This softening is substantially smaller than that previously reported. In previous studies, softening was observed in a multilayer system at $d_{\mathrm{Cu}} / d_{\mathrm{Nb}}=1$, whereas our results for the same system show that the stiffness is almost independent of the bilayer thickness. The elastic stiffness of the multilayers is, thus, smaller than their bulk stiffness because of the softening of each layer.

In the present study, thickness of each layer in multilayers $(\sim 1 \mathrm{~nm})$ is significantly smaller than that of monolayer films we measured $(\sim 60 \mathrm{~nm})$. One may consider that elastic stiffness of the monolayer films should not be used to estimate the elastic stiffness of multilayers, because the thickness is quite different each other and the stiffness of monolayer films may depends on the film thickness. However, measurement of film stiffness at around $1 \mathrm{~nm}$ is quite difficult. For example, regarding picosecond ultrasonics, when the film thickness is smaller than about $40 \mathrm{~nm}$, resonant vibration is observed, instead of the propagation of the acoustic pulse in the thickness direction, and elastic stiffness can be determined from resonance frequency. ${ }^{19)}$ However, detection of the resonant vibration becomes difficult below $10 \mathrm{~nm}$; the measurable minimum thickness depends on material. Therefore, the thickness dependence and interface effects are often evaluated indirectly by measuring elastic stiffness of multilayers with various configurations. Under the circumstance, we observed that the macroscopic stiffness of multilayers with different thickness ratios can be reproduced by using the elastic stiffness of thicker monolayer films. This result indicates that elastic stiffness of thin films is insensitive to the thickness even at around $1 \mathrm{~nm}$ thickness. 
In previous studies using the Brillouin scattering, ${ }^{1,2)}$ multilayers consisting of more than a few hundred layers were prepared. During the deposition of the large number of the layers, coherency at the interface might be disturbed, possibly resulting in a defective structure and in less interface stiffness. In the present study, the multilayers comprised 80 or fewer layers. The difference in the number of layers is a possible cause of the nonsoftening at interfaces, in addition to the uncertainty in measurement. In the study using the picosecond ultrasonis, notable softening is observed in the multilayers with less ordered structures as described above. Therefore, we speculate that the effect of interface stiffness on macroscopic stiffness is essentially small and that softening in each layer is a dominant factor. Under this interpretation, we can estimate macroscopic stiffness by measuring the elastic stiffness of monolayers of the constituting materials without measuring the macroscopic elastic stiffness of multilayers.

\section{Conclusions}

In this study, the elastic stiffness of $\mathrm{Cu} / \mathrm{Co}, \mathrm{Cr} / \mathrm{Fe}$, and $\mathrm{Nb} / \mathrm{Cu}$ multilayers was measured using picosecond ultrasonics. The measured elastic stiffness was smaller than the macroscopic stiffness calculated from the bulk stiffness, and it was similar to those calculated from the film stiffness. Compared with the previously reported experimental results, the dependence of elastic stiffness on the number of interfaces was minor in $\mathrm{Nb} / \mathrm{Cu}$ multilayers. In addition, the results experimentally revealed that macroscopic stiffness is insensitive to the number of interfaces (bilayer thickness) and that the softening of each layer is the dominant cause of the lesser macroscopic stiffness.

\section{Acknowledgment}

The authors wish to thank S. Akai for his contributions to measurements. 


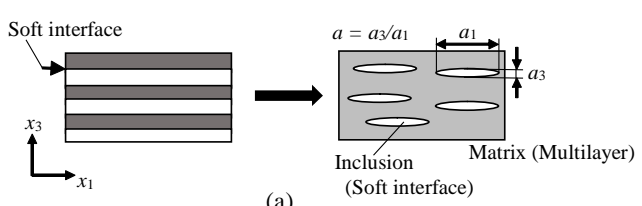

(a)

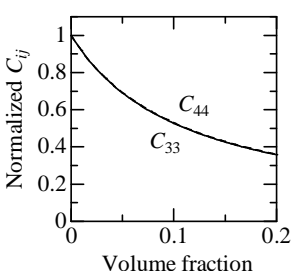

(b)

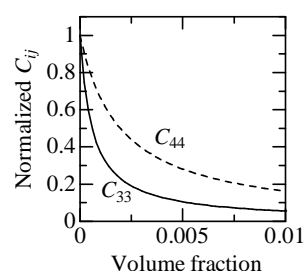

(c)

Fig. A.1. Elastic stiffness of a composite composing an isotropic matrix and isotropic penny shape inclusions calculated using the micromechanics. (a) The considered composite model. Elastic stiffness of the matrix is $C_{11}=200 \mathrm{GPa}$ and $C_{44}=50 \mathrm{GPa}$. Elastic stiffness of the inclusions is $C_{11}=20 \mathrm{GPa}$ and $C_{44}=5$ $\mathrm{GPa}$ in (b), and is $C_{11}=0.0001 \mathrm{GPa}$ and $C_{44}=0 \mathrm{GPa}$ in (c). Aspect ratio $a$ of the inclusions is 0.01. Curves of $C_{33}$ and $C_{44}$ are almost overlapped in (b).

\section{Appendix: Sensitivity of the longitudinal-wave elastic stiffness to the interface stiffness}

Sensitivity of the longitudinal-wave elastic stiffness to the interface stiffness is discussed here. For this discussion, we propose a composite material. The composite composes an isotropic matrix and isotropic penny-shape $\left(a_{1}=a_{2}>>a_{3}\right)$ inclusions (Fig. A·1(a)). This composite is a model of a multilayer that contains soft interfaces. Two sets of elastic stiffness were assumed for the inclusions. Elastic stiffness of the first set is one tenth of that of the matrix. Elastic stiffness of the second set is significantly smaller than that of the matrix, reproducing thin voids at interfaces. Elastic stiffness of the composite was calculated using micromechanics, and the results are shown in Fig. A.1. Detail of the calculation procedure is described elsewhere. ${ }^{41)}$ The calculation results indicate that the sensitivity of $C_{33}$ to the interface softening is comparable to or larger than that of $C_{44}$; the Rayleigh-wave velocity highly depends on $C_{44}$. Thus, softening at interfaces is detectable by measuring $C_{33}$. 


\section{References}

1) A. Kueny, M. Grimsditch, K. Miyano, I. Banerjee, C. M. Falco, and I. K. Schuller, Phys. Rev. Lett. 48, 166 (1982).

2) J. A. Bell, W. R. Bennett, R. Zanoni, G. I. Stegeman, C. M. Falco, and C. T. Seaton, Solid State Commun. 641339 (1987).

3) R. Danner, R. P. Huebener, C. S. L. Chun, M. Grimsditch, and I. K. Schuller, Phys. Rev. B 33, 3696 (1986).

4) G. Richardson, J. L. Makous, H. Y. Yu, and A. S. Edelstein, Phys. Rev. B 45, 114 (1992).

5) G. Carlotti, G. Gubbiotti, L. Pareti, G. Socino, and G. Turilli, J. Magn. Magn, Mater. 165, 424 (1997).

6) U. Harms and R. B. Schwarz, Phys. Rev. B 65, 085409 (2002).

7) H. Huang and F. Spaepen, Acta Mater. 48, 3261 (2000).

8) B. M. Clemens and G. L. Eesley, Phys. Rev. Lett. 61, 2356 (1988).

9) E. E. Fullerton, I. K. Schuller, F. T. Parker, K. A. Svinarich, G. L. Eesley, R. Bhadra, and M. Grimsditch, J. Appl. Phys. 73, 7370 (1993).

10) B. Perrin, B. Bonello, J. -C. Jeannet, and E. Romater, Physica B 219\&220, 681 (1996).

11) N. Nakamura, A. Uranishi, M. Wakita, H. Ogi, and M. Hirao, Jpn. J. Appl. Phys. 49, 07HB04 (2010).

12) N. Nakamura, H. Ogi, T. Yasui, M. Fujii, and M. Hirao, Phys. Rev. Lett. 99, 035502 (2007).

13) P. Baumgart, B. Hillebrands, R. Mock, G. Güntherodt, A. Boufelfel, and C. M. Falco, Phys. Rev. B 34, 9004 (1986).

14) J. A. Bell, W. R. Bennett, R. Zanoni, G. I. Stegeman, C. M. Falco, and F. Nizzoli, Phys. Rev. B. 35, 4127 (1987).

15) J. R. Dutcher, S. Lee, J. Kim, G. I. Stegeman, and C. M. Falco, Phys. Rev. Lett. 65, 1231 (1990).

16) B. Hillebrands, P. Krams, K. Spörl, and D. Weller, J. Appl. Phys. 69, 938 (1991).

17) G. Carlotti, D. Fioretto, G. Socino, B. Rodmacq, and V. Pelosin, J. Appl. Phys. 71, 4897 (1992).

18) D. Wolf and J. F. Lutsko, Phys. Rev. Lett. 60, 1170 (1988).

19) H. Ogi, M. Fujii, N. Nakamura, T. Yasui, and M. Hirao, Phys. Rev. Lett. 98, 195503 (2007). 
20) J. R. Dutcher, S. Lee, C. D. England, G. I. Stegeman, and C. M. Falco, Mater. Sci. Eng. A 126, 13 (1990).

21) C. Thomsen, H. T. Grahn, H. J. Maris, and J. Tauc, Phys. Rev. B 34, 4129 (1986).

22) N. Nakamura, H. Ogi, T. Shagawa, and M. Hirao, Appl. Phys. Lett. 92, 141901 (2008).

23) N. Nakamura, Y. Kake, H. Ogi, and M. Hirao, J. Appl. Phys. 108, 043525 (2010).

24) N. Nakamura, A. Uranishi, M. Wakita, H. Ogi, M. Hirao, and M. Nishiyama, Appl. Phys. Lett. 98, 101911 (2011).

25) W. Chen, Y. Lu, H. J. Maris, and G. Xiao, Phys. Rev. B 50, 14506 (1994).

26) L. Belliard, A. Huynh, B. Perrin, A. Michel, G. Abadias, and C. Jaouen, Phys. Rev. B 80, 155424 (2009).

27) H. Ogi, T. Shagawa, N. Nakamura, and M. Hirao, Appl. Phys. Express 2, 105001 (2009).

28) H. Ogi, H. Ledbetter, S. Kim, and M. Hirao, J. Acoust. Soc. Am. 106, 660 (1999).

29) M. J. O’Neil, P. E. Heckelman, P. H. Dobbelaar, K. J. Roman, C. M. Kenny, and L. S. Karaffa, The Merck index (The Royal Society Chemistry, Cambridge, 2013).

30) P. E. Armstrong, J. M. Dickinson, and H. L. Brown, Trans. Metall. Soc. AIME 236, 1404 (1966).

31) H. J. McSkimin, J. Appl. Phys. 26, 406 (1955).

32) L. G. Parratt, Phys. Rev. 95, 359 (1954).

33) B. P. Tonner, Z. L. Han, and J. Zhang, Phys. Rev. B 47, 9723 (1993).

34) H. T. Grahn, H. J. Maris, J. Tauc, and B. Abeles, Phys. Rev. B 38, 6606 (1988).

35) H. J. Leamy and H. Warlimont, Phys. Stat. Sol. 37, 523 (1970).

36) C. A. Rotter and C. S. Smith, J. Phys. Chem. Solids 27, 267 (1966).

37) D. I. Bolef and J. De Klerk, Phys. Rev. 129, 1063 (1963).

38) N. Nakamura, H. Ogi, and M. Hirao, Phys. Rev. B 77, 245416 (2008).

39) N. Nakamura, H. Ogi, H. Nitta, H. Tanei, M. Fujii, T. Yasui, and M. Hirao, Jpn. J. Appl. Phys. 45, 4580 (2006).

40) N. Nakamura, H. Ogi, and M. Hirao, Acta Mater. 52, 765 (2004).

41) H. Ogi, G. Shimoike, M. Hirao, K. Takashima, and Y. Higo, J. Appl. Phys. 91, 4857 (2002). 